\title{
Linx
}

Revue des linguistes de l'université Paris X Nanterre

$45 \mid 2001$

Invariants et variables dans les langues. Études typologiques

\section{Deux types de prédicats complexes dans les langues romanes}

Anne Abeillé et Danièle Godard

\section{(2) OpenEdition}

Journals

Édition électronique

URL : http://journals.openedition.org/linx/838

DOI : $10.4000 / \operatorname{lin} x .838$

ISSN : 2118-9692

Éditeur

Presses universitaires de Paris Nanterre

Édition imprimée

Date de publication : 1 décembre 2001

Pagination : 167-175

ISSN : 0246-8743

Référence électronique

Anne Abeillé et Danièle Godard, "Deux types de prédicats complexes dans les langues romanes », Linx [En ligne], 45 | 2001, mis en ligne le 25 juin 2012, consulté le 20 avril 2019. URL : http:// journals.openedition.org/linx/838; DOI : 10.4000/linx.838

Ce document a été généré automatiquement le 20 avril 2019

Département de Sciences du langage, Université Paris Ouest 


\title{
Deux types de prédicats complexes dans les langues romanes ${ }^{1}$
}

\author{
Anne Abeillé et Danièle Godard
}

1 Les prédicats complexes romans (auxiliaires, verbes restructurants, causatifs, attributifs) qu'on peut définir formellement par la propriété de «montée » des pronoms clitiques, sont souvent considérés comme formant un type, monoclausal analytique, à mi-chemin entre le type à construction biclausale (ex. les causatifs anglais) et le type monoclausal synthétique (ex. le causatif bantou).

2 Nous voulons montrer que, pour les auxiliaires et les verbes dits "restructurants " (modaux, aspectuels), si l'on prend en compte les structures syntaxiques, on a affaire à au moins deux types distincts, qu'on retrouve dans des langues non apparentées.

3 Nous rappellerons d'abord (\$1) les propriétés communes de monoclausalité, avant de présenter les propriétés contrastives (insertion d'adverbes et portée de la coordination) pour les auxiliaires de temps (\$2.1) et pour les verbes « restructurants» (\$2.2). Les langues considérées ici sont le français $(\mathrm{F})$, l'espagnol (E), le roumain (R) et l'italien (I)².

\section{Les propriétés communes de monoclausalité}

4 Les séquences verbales regroupées sous le terme de "prédicat complexe » ont dans les langues romanes un certain nombre de propriétés syntaxiques, morphologiques et sémantiques bien connues: le placement des pronoms clitiques compléments peut se faire sur le premier verbe (même s'il s'agit d'un complément sous-catégorisé par un verbe suivant), le premier verbe a une contribution sémantique «faible» (temps, aspect, modalité...), seul le premier verbe est fléchi (les autres sont à l'infinitif, au gérondif ou au participe). Nous allons privilégier ici les aspects syntaxiques. 


\subsection{La montée des pronoms clitiques}

5 La «montée » des clitiques compléments est obligatoire pour les auxiliaires de temps: E haber, F avoir, être, I avere, essere, R a avea, a fi, aş, voi.

\begin{tabular}{|l|l|l|l|}
\hline$(1)$ & F & a. & Jean ne l'a pas vu/ * n'a pas le-vu \\
\hline & I & b. & Giovanni non l'ha veduto/ * non ha lo-veduto/ * vedutolo \\
\hline & E & c. & Juan no lo ha visto/ * non ha lo visto/ * non ha vistolo \\
\hline & R & d. & Ion nu l-a văzut/ * nu a-l-văzut/ * nu a văzutu-l \\
\hline
\end{tabular}

6 Elle est optionnelle pour les verbes "restructurants » (modaux, aspectuels), sauf en roumain où elle est obligatoire: I dovere, cominciare, potere, volere, sapere..., E deber (de), haber de, poder, querer..., R a putea (+inf), a avea de (+supin). S'il y a optionalité, le placement des clitiques compléments est possible sur chacun des verbes, avec le même sens (et parfois des différences stylistiques ou régionales). Nous contrastons pour l'italien les restructurants optionnels (volere) et les non-restructurants (desiderare) :

\begin{tabular}{|l|l|l|l|}
\hline (2) & I & a. & Giovanni vuole / desidera mangiarle. \\
\hline & & a'. & Giovanni le vuole / * desidera mangiare. \\
\hline & E & b. & Juan quiere comerlas/ las quiere comer. \\
\hline & & & Jean veut/désire les manger \\
\hline \hline & R & c. & Ion o poate vedea./ ${ }^{*}$ poate vedea-o. $/{ }^{*}$ poate o vedea. \\
\hline & & & Jean peut la voir \\
\hline
\end{tabular}

7 Bien qu'il y ait un groupe commun de V restructurants, il y a aussi des spécialisations lexicales (pour les langues, et les locuteurs).

\subsection{Le médio-passif}

8 Comme il a été noté par (Rizzi 1982, Aissen \& Perlmutter 1983), le médio-passif (ou se moyen) peut s'appliquer au premier verbe, s'il s'agit d'un verbe restructurant. Il s'applique ainsi à I potere, E poder, et à R a putea,mais pas à I affirmare, ou à $\mathrm{E}$ afirmar qui ne sont pas les verbes permettant la « montée » du clitique :

\begin{tabular}{|l|l|l|l|}
\hline (3) & I & a. & Queste camicie si possono / * affirmano stirare facilemente. \\
\hline \hline & E & b. & Estas camisas se pueden / * afirman planchar fácilmente. \\
\hline
\end{tabular}


Cămaşile acestea se pot călca uşor.

9 Ce type de médio-passif ne s'applique pas si l'infinitif porte un clitique, ce qui indique bien la corrélation avec la montée des clitiques :

\begin{tabular}{|l|l|l|l|}
\hline (4) & I & a. & Queste case gli si vogliono vendere a caro prezzo. \\
\hline & & & lit. Ces maisons leur se veulent vendre à cher prix \\
\hline & & & On veut leur vendre cher ces maisons \\
\hline & & a'. & $*$ Queste case si vogliono vendergli a caro prezzo. \\
\hline & E & b. & Estas casas se le quieren vender al general. \\
\hline & & & lit. Ces maisons se lui veulent vendre au général \\
\hline & & & On veut vendre ces maisons au général. \\
\hline & & b'. & $*$ Estas casas se quieren venderle al general. \\
\hline
\end{tabular}

10 Ceci montre qu'en $(4 a, b)$ les deux verbes ne partagent pas seulement les compléments cliticisés, mais aussi l'objet direct (qui devient sujet du premier verbe au médio passif). Cela est confirmé par la propriété suivante.

\subsection{Les constructions infinitives à objet manquant}

11 Comme noté par (Huot 1981, Rizzi 1982), les constructions infinitives à objet manquant sont des cas de dépendances bornées, dans les langues romanes, c'est-à-dire que l'infinitif dont l'objet manque ne doit pas être enchâssé :

\begin{tabular}{|l|l||l|l|}
\hline$(5)$ & F & a. & Un texte impossible à comprendre rapidement \\
\hline & I & b. & Un testo impossibile da capire rapidamente \\
\hline & E & C. & Un texto imposible de entender rápidamente \\
\hline & & & \\
\hline (6) & F & a. & * Un texte impossible à exiger de comprendre rapidement \\
\hline & I & b. & $*$ Un testo impossibile da esigere di capire rapidamente \\
\hline & E & C. & $*$ Un texto imposible de exigir entender rápidamente \\
\hline
\end{tabular}

Les seuls cas où le verbe à objet manquant n'est pas le premier infinitif sont précisément les cas de prédicat complexe (autorisant la « montée » des clitiques) : 


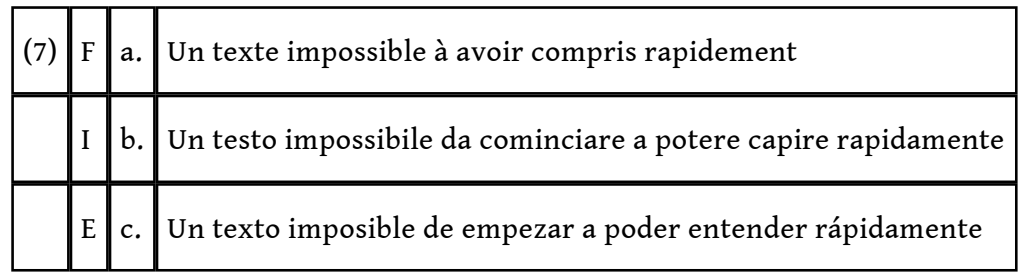

13 En (7) c'est l'objet attendu par compris, capire ou entender, qui manque (et qui dénote le même objet que le $\mathrm{N}$ auquel s'adjoint l'adjectif). Compte tenu de la contrainte bloquant les phrases de type (6), cela signifie que F avoir, I cominciare, potere, E empezar ou poder doivent être analysés comme attendant le même objet que le verbe qui les suit en (7).

Ces différentes propriétés indiquent que les prédicats complexes correspondent à une phrase simple, où les séquences de verbes forment un seul domaine pour la souscatégorisation (on peut parler de partage d'arguments ou de partage de valence). Mais elles ne disent rien de la structure syntaxique de ces phrases. C'est à cet aspect structural que nous allons maintenant nous attacher, en utilisant d'autres propriétés, qui sont des critères de structure, indépendamment de telle ou telle théorie syntaxique.

\section{Les propriétés structurales contrastives}

15 Nous avons a priori pour ces constructions trois structures possibles: la structure à complément syntagmatique (ou structure hiérarchique: I), la structure à sousconstituant verbal (ou complexe verbal : II) et la structure "plate» où verbes lexicaux et compléments sont tous au même niveau (III).
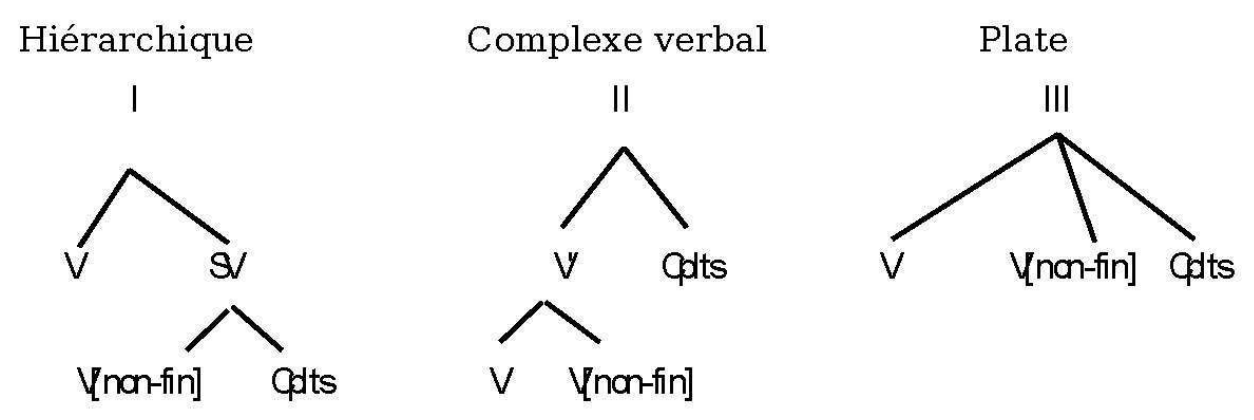

16 La première structure est difficilement compatible avec les propriétés de monoclausalité vues supra (il faut faire la différence avec les verbes non restructurants) et directement contredite par les propriétés d'extraction que nous allons maintenant présenter.

Nous montrerons que les langues romanes se répartissent, pour les prédicats complexes, entre les deux autres structures, selon leur réaction aux tests d'insertion et de coordination, que nous utilisons comme indices structuraux. La structure à complexe verbal force une quasi adjacence entre les deux verbes, et prévoit qu'il sera difficile d'insérer des adverbes variés. De même, il sera difficile, avec cette structure, de coordonner des séquences de verbes non finis avec leurs compléments sans répéter le premier verbe, car il s'agirait de coordonner des séquences de constituants de niveau différent. 
18 La structure plate traite le second verbe comme un complément lexical (« nu »), au même niveau que les autres compléments, et autorise aussi bien les insertions que la coordination de séquences verbe-complément, sans qu'il soit nécessaire de répéter le premier verbe.

\subsection{Les auxiliaires de temps}

Contrairement à ce qu'on observe en anglais (avec VP fronting ou VP ellipsis), on ne peut jamais extraire ou élider la séquence participe + Compléments, dans les langues romanes actuelles :

\begin{tabular}{|l|l|l|l|}
\hline (8) & F & a. & * Ce que Jean a, c'est lu ce livre. \\
\hline & & a'. & * Jean a lu ce livre mais Marie n'a pas. \\
\hline & E & b. & * Lo que Juan ha es leído este libro. \\
\hline & & b' & * Juan ha leído eso y Marta ha también. \\
\hline & I & c. & $*$ È letto questo libro che Giovanni ha. \\
\hline & & '' & $*$ Paolo ha letto questo libro ma Maria non ha. \\
\hline & R & d. & $*$ Ceea ce a este citit această carte. \\
\hline & & d' & $*$ Ion a citit o carte, dar Maria nu a. \\
\hline
\end{tabular}

20 La coordination de participes, sans répétition de l'auxiliaire, est toujours possible, ce qui montre bien que les deux verbes sont deux mots distincts, combinés en syntaxe, et non en morphologie :

\begin{tabular}{|l||l|l|l|}
\hline (9) & F & a. & Jean a acheté et lu le livre. \\
\hline & I & b. & Giovanni ha comprato e letto il libro. \\
\hline & E & c. & Juan ha comprado y leído este libro. \\
\hline & R & d. & Ion a cumpărat şi citit această carte. \\
\hline
\end{tabular}

21 En revanche, pour ce qui est de la coordination de séquences participe + Compléments, le contraste est net entre F et I, qui l'autorisent, et $\mathrm{E}$ et $\mathrm{R}$ qui la refusent ${ }^{3}$ :

\begin{tabular}{|l|l|l|l|}
\hline$(10)$ & F & a. & Jean a acheté ce livre et lu le premier chapitre. \\
\hline & I & b. & Giovanni ha sbattutto gli albumi e fatto la crema. \\
\hline
\end{tabular}




\begin{tabular}{|l|l|l|l|}
\hline & & & Giovanni a battu les œufs et fait la crème \\
\hline & E & c. & * Juan ha comprado este libro y leído el primer capitulo. \\
\hline & & c.' & Juan ha comprado este libro y ha leído el primer capitulo. \\
\hline & R & d. & * Ion a cumpărat această carte şi citit primul capitol. \\
\hline & & d'. & Ion a cumpărat această carte şi a citit primul capitol. \\
\hline & & & Juan/Ion a acheté ce livre et a lu le premier chapitre. \\
\hline
\end{tabular}

L'insertion d'adverbes entre les deux verbes est naturelle en $\mathrm{F}$ et I, et difficile en $\mathrm{E}$ et $\mathrm{R}$. En espagnol, elle est prohibée par les grammaires normatives, et limitée dans les textes à quelques adverbes (casi, ya), adjoints au V lexical. En roumain (cf. Dobrovie-Sorin 1994), elle se limite aux cinq clitiques adverbiaux (mai, şi, tot, prea, cam).

\begin{tabular}{|l|l|l|l|}
\hline (11) & F & a. & Jean lui a généreusement donné des disques. \\
\hline & I & b. & Giovanni gli ha generosamente dato dei dischi. \\
\hline & E & c. & * Los niños no habían todavía aprendido a hablar. \\
\hline & & & Les enfants n'ont pas encore appris à parler \\
\hline & & c' & ? Los niños habían casi aprendido a hablar. \\
\hline & & & Les enfants ont presque appris à parler \\
\hline \hline & R & d. & * Maria îi-a atent ascultat. \\
\hline & & & Marie les a attentivement écoutés \\
\hline & & d' & Nu l-am mai văzut. \\
\hline & & & Je ne l'ai jamais vu \\
\hline
\end{tabular}

On a donc une corrélation remarquable entre les propriétés de coordination et celles d'insertion. Ceci s'explique à partir du moment où l'on attribue la structure à complexe verbal (II) à $\mathrm{E}$ et $\mathrm{R}$, et la structure plate (III) à $\mathrm{F}$ et I. 
E,R : Structure II

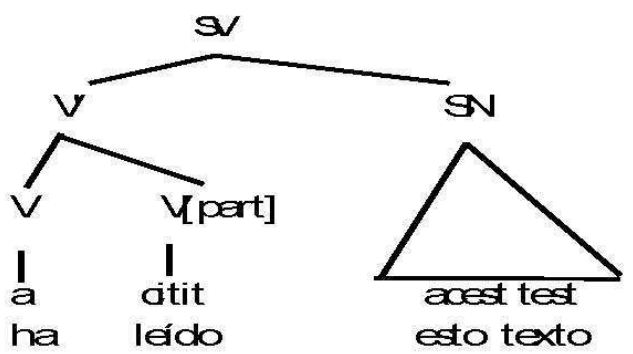

F ,I : Structure III

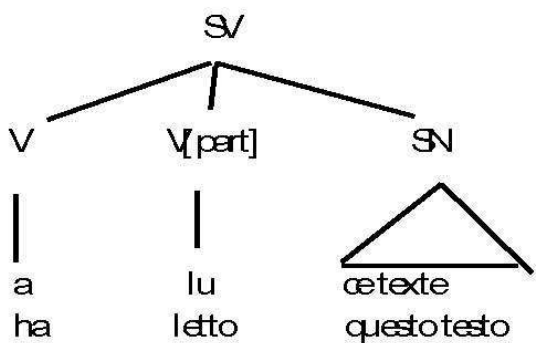

\begin{tabular}{|l|l|l|l|}
\hline (13) & I & a. & La tua disavventura, è proprio a raccontarla a Mario che ho cominciato. \\
\hline & & & lit. Ta mésaventure, c'est justement à la raconter à Mario que j'ai commencé \\
\hline & & a'. & * La tua disavventura, è proprio a raccontare a Mario che l'ho cominciata. \\
\hline & E & b. & Lo que Juan quiere es leer mi libro. \\
\hline & & & Ce que Jean veut, c'est lire mon livre \\
\hline & & b'. & * Lo que Juan lo quiere es leer. \\
\hline & R & c. & * Citi cartea nu am putut. \\
\hline & & & Lire le livre, je n'ai pas pu \\
\hline
\end{tabular}

Dans la structure plate, la coordination de séquences participe + compléments se fait de la même façon que pour les coordinations de séquences de compléments d'un même verbe (cf. 12a). Avec la structure à complexe verbal, ce type de coordination est exclu, comme sont exclues les coordinations de séquences de constituants de niveau différent (cf. 12b) :

\begin{tabular}{|l|l|l|}
\hline$(12)$ & a. & Jean donne [des livres ] [à son fils] et [des disques ] [à sa fille ] \\
\hline \hline & b. & * Jean donne [un livre [sur Proust ]] [à son fils] et [sur Camus ] [à sa fille] \\
\hline
\end{tabular}

Le même contraste structural entre langues se retrouve avec les verbes dits restructurants (modaux, aspectuels).

\subsection{Les verbes « restructurants »}

Lorsque les clitiques sont montés, les verbes modaux, et aspectuels n'autorisent pas l'extraction de la séquence infinitif + compléments (cf. Rizzi 1982) :

Si la structure hiérarchique (I) est bien adaptée à ces verbes, quand le clitique reste sur l'infinitif $(13 a, b)$, elle n'est pas appropriée, en revanche, dans les constructions à clitique « monté » qui nous intéressent (13a',b',c). 
Examinons maintenant les propriétés d'insertion et de coordination. Le français n'ayant plus aujourd'hui de verbes restructurants, le contraste est désormais entre italien et espagnol. Dans les constructions à clitique monté, la coordination de séquences infinitif + compléments (sans répétition du modal) est naturelle en italien et en roumain, mais difficile en espagnol :4

\begin{tabular}{|l|l|l|l|}
\hline (14) & I & a. & Lo vuole comprare domani e dare a Giovanni per il suo compleanno. \\
\hline & & & lit. Le veut acheter demain et donner à G pour son anniversaire \\
\hline & & & Il veut l'acheter demain et le donner à G pour son anniversaire \\
\hline & & a'. & Paola gli deve telefonare domani e parlare del problemo. \\
\hline & & & lit. Paola lui doit téléphoner demain et parler du problème \\
\hline & & & Paola doit lui téléphoner demain et lui parler du problème \\
\hline & E & b. & Juan quiere comprarlo para él y leerlo en seguida. \\
\hline & & & Juan veut l'acheter pour lui et le lire aussitôt \\
\hline & & b'. & *Juan lo quiere comprar para él y leer en seguida. \\
\hline & & & lit. Juan le veut acheter pour lui et lire tout de suite \\
\hline & R & c. & Ion poate cumpăra această carte şi citi primul capitol. \\
\hline & & & Ion peut acheter ce livre et lire le premier chapitre \\
\hline
\end{tabular}

Il est remarquable que le roumain se comporte ici comme l'italien et non comme l'espagnol.

30 La corrélation avec l'insertion d'adverbes variés entre les deux verbes (avec clitique monté) est la même que précédemment. Les langues autorisant la portée large du modal (avec clitique monté) sur une coordination de séquences infinitives sont aussi celles qui autorisent l'insertion d'adverbes, c'est-à-dire ici l'italien et le roumain. Comme pour les auxiliaires, l'insertion d'adverbes est limitée en espagnol (avec clitique monté) :

\begin{tabular}{|l|l|l|l|}
\hline (15) & I & a. & Giovanni gli vuole generosamente dare dei dischi. \\
\hline & & & lit. Giovanni lui veut généreusement donner des disques \\
\hline & E & b. & * Juan lo quiere a menudo leer. \\
\hline & & & lit. Juan le veut souvent lire \\
\hline & & b'. & Juan quiere a menudo leerlo. \\
\hline
\end{tabular}




\begin{tabular}{|l|l|l|l|}
\hline & & b ». & ? Juan lo puede casi leer. \\
\hline & R & c. & Maria îi-poate eventual asculta. \\
\hline & & & lit. Maria les peut éventuellement écouter \\
\hline
\end{tabular}

Pour les constructions sans montée de clitiques (F, E et I), nous avons donc la structure hiérarchique (I). Pour les constructions à montée de clitiques, nous proposons la structure à complexe verbal (II) pour les verbes restructurants de l'espagnol, et la structure plate (III) pour ceux de l'italien et pour le modal roumain ${ }^{5}$.

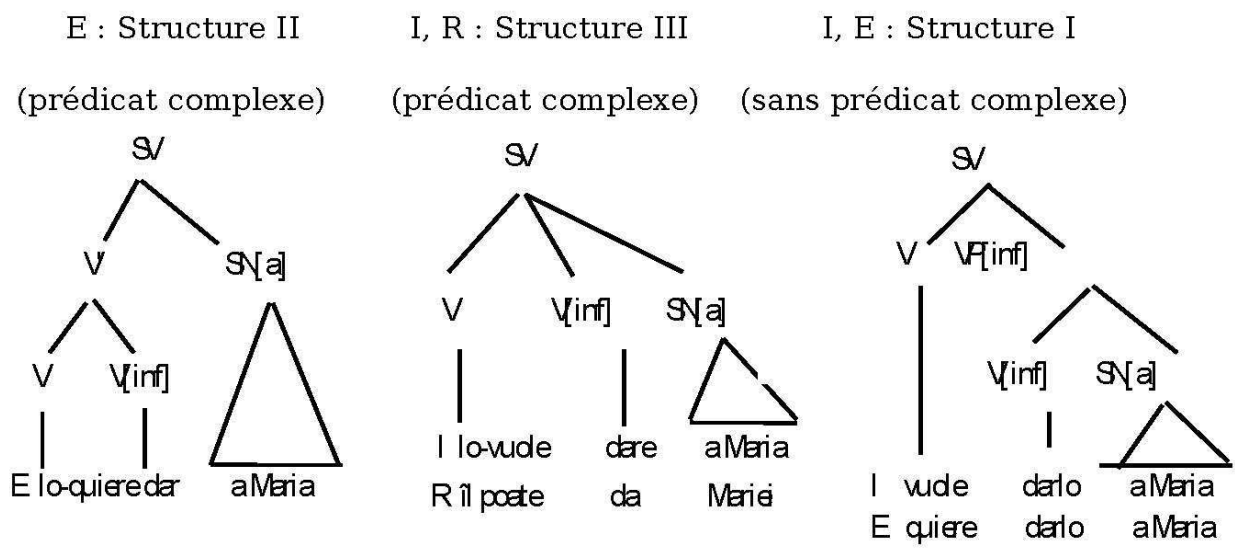

Si l'on prend en compte les constructions causatives (Abeillé et al. 1997, Abeillé et Godard à paraître), le contraste structural est le même entre d'une part français et italien (structure plate) et d'autre part espagnol et catalan (structure à complexe verbal). Il est remarquable que les langues tendent à choisir un seul type de structure pour toutes ses constructions à prédicat complexe (auxiliaires, modaux, causatifs) le roumain étant ici exceptionnel ${ }^{6}$.

\section{Conclusion}

Si l'on prend au sérieux la notion de structure de constituants, on doit définir deux types syntaxiquement distincts, pour les prédicats complexes romans :

- le type A, qui regroupe l'espagnol et les auxiliaires de temps du roumain, ainsi que le catalan, avec constitution d'un complexe verbal (sous-constituant),

- le type B, qui regroupe l'italien, le français et les modaux roumains, ainsi que l'occitan et le portugais, caractérisé par une structure plate, où les verbes lexicaux sont tous au même niveau que les compléments.

Ces deux structures se retrouvent dans d'autres langues, où la notion de prédicat complexe est utilisée pour le partage d'arguments entre verbes (sans qu'il y ait forcément cliticisation). Le type A se retrouve pour les auxiliaires coréens (Chung 1998) et les séquences de verbes en fin de phrase en allemand. Le type B se retrouve avec certains auxiliaires polonais (Borsley 1999).

Une autre conclusion est qu'il semble important, sur le plan théorique, de distinguer la représentation de la valence (ou de la sous-catégorisation) de celle de la structure de 
constituants, comme le font des modèles comme LFG ou HPSG. Les prédicats complexes des langues romanes se comportent de la même façon pour ce qui est du partage de valence, mais de façon différente pour ce qui est de la structure syntaxique. Une explication purement structurale du placement des clitiques ne peut donc être satisfaisante.

\section{BIBLIOGRAPHIE}

ABEILLÉ, A., GODARD, D. (1996). La Complémentation des auxiliaires en français, Langages 122, pp. 32-61.

ABEILLÉ, A., GODARD, D., MILLER, P. (1997). Les Constructions causatives en français : un cas de compétition syntaxique, Langue française 115, pp. 62-74.

ABEILlÉ, A., GODARD, D., SAG I. A. (1998). Two Kinds of Composition in French Complex Predicates. in E. Hinrichs et al (eds), Complex predicates in non-derivational syntax, New York : Academic Press, pp1-41.

ABEILLÉ, A., GODARD, D., (2000). Varieties of ESSE in Romance Languages. in D. Flickinger et al. (eds), Berkeley Formal Grammar Conference, Stanford : CSLI Publications.

ABEILLÉ, A., GODARD, D., (à paraître). Les Prédicats complexes in D. Godard (ed). Les Langues romanes. Problèmes de la phrase simple, Paris : CNRS Editions.

AISSEN, J., PERlmutTeR, D. (1983). Clause Reduction in Spanish. in D. Perlmutter (ed), Studies in relational grammar 1, Chicago : University of Chicago Press, pp. 360-403.

BARBU, A.M. (1998). Complexul verbal. MS (à paraître Studii si cercetari Lingvistice).

BORSLEY, R. (1999). Auxiliaries, Verbs and Complementizers in Polish, in R. Borsley, A.

Przepiorkowski (eds), Slavic in HPSG, Stanford : CSLI, pp. 29-60.

BOSQUE, I., DELMONTE, V. (eds). (1999). Gramática descriptiva de la lengua española, Madrid: Espasa Calpe. 3 vol.

CHUNG C., (1998). Argument Composition and Long Distance Scrambling in Korean : an extension of the complex predicate analysis, in E Hinrichs et al. (eds), Complex predicates in non derivational syntax, New York: Academic Press. pp 159-220.

DOBROVIE-SORIN, C. (1994). The Syntax of Romanian, Berlin, New-York : Mouton de Gruyter.

нUот H. (1981). Constructions infinitives du français : le subordonnant de, Genève : Droz.

MILLER, P., SAG, I.A. (1997). French Clitic Movement without Clitics or Movement. Natural Language and Linguistic Theory 15, pp. 573-639.

MONACHESI, P. (1999a). A Lexical Approach to Italian Cliticization. Stanford : CSLI.

MONACHESI, P. (1999b) The Syntactic Structure of Romanian Auxiliary (and Modal) Verbs. in G. Bouma et al. (eds), Constraints on Resources in Natural Language Syntax and Semantics, Stanford : CSLI, pp. 101-118. 
RENZI, L., SALVI, G. (eds). (1988-91). Grande Grammatica italiana di consultazione, Bologne : il Mulino.

RIZZI, L. (1982). Issues in Italian syntax. Dordrecht : Foris.

SUÑER, M. (1987). Haber + past participle, Linguistic Inquiry 18, pp. 683-690.

\section{NOTES}

1. Nous remercions pour leurs commentaires et leurs jugements: A-M. Barbu, C. BlancheBenveniste, D. Creissels, R. Delmonte, J. Delofeu, B. Laca, A. Mari, P. Monachesi, F. Namer, A. Rouveret, L. Tasmowski, L. Tovena. Une version plus étendue de ce travail doit paraitre dans le livre édité par D. Godard aux éditions du CNRS.

2. Pour le portugais, le catalan et l'occitan, que nous rangeons dans le groupe français-italien, voir Abeillé et Godard (à paraître). Pour des raisons de place, nous laissons de côté les constructions attributives, qui sont présentées dans Abeillé et Godard $(1996,2000)$ et (à paraître).

3. En espagnol, ce type de coordination est possible avec certains compléments «nus» (noms sans déterminants) :

E a. Pablo ha comido galletas y bebido leche.

a'. * Pablo ha comido dos galletas y bebido toda la leche.

On peut montrer que ces noms sont en fait adjoints à $\mathrm{V}$, ce qui est compatible avec la structure à complexe verbal.

4. Comme avec les auxiliaires, la coordination est meilleure si le complément nominal est «nu» :

$\mathrm{E}$ b. Juan le quiere comprar libros y dar discos.

Lit. Juan lui veut acheter livres et donner disques

b'. *Juan le quiere comprar un libro y dar un disco.

Juan veut lui acheter des livres et lui donner des disques

5. Dans les arbres, nous indiquons les clitiques comme affixés au verbe, suivant en cela (Miller et Sag 1997) mais notre analyse est indépendante du traitement des clitiques en morphologie ou en syntaxe.

6. Le roumain utilise les deux structures, mais avec une préférence pour la structure à complexe verbal, puisque l'usage du modal avec l'infinitif y est résiduel. D'autre part, les verbes attributifs ont les deux mêmes structures dans toutes les langues romanes (structure hiérarchique et structure plate).

\section{AUTEURS}

\section{ANNE ABEILLÉ}

LLF, UFRL, Case 7003, Université Paris 7, 2 place Jussieu, 75005 Paris

abeille@linguist.jussieu.fr

\section{DANIÈLE GODARD}

LLF, UFRL, Case 7003, Université Paris 7, 2 place Jussieu, 75005 Paris

dgodard@linguist.jussieu.fr 